\title{
Re-absorption and nonradiative energy transfer in vibronic laser gain media
}

\author{
Jonathan W. Evans $\odot,{ }^{\text {a,* } *}$ Thomas R. Harris, ${ }^{\text {a,b }}$ Eric J. Turner, ${ }^{\text {a,c }}$ \\ Martin M. Kimani, ${ }^{\text {a,c }}$ J. Matthew Mann, ${ }^{\text {a Ronald W. Stites, }}{ }^{\text {a }}$ \\ Gary Cook, ${ }^{a}$ and Kenneth L. Schepler ${ }^{\text {d }}$ \\ ${ }^{a}$ Air Force Research Laboratory, Sensors Directorate, Wright-Patterson Air Force Base, Ohio, \\ United States \\ ${ }^{\mathrm{b}}$ Azimuth Corporation, Fairborn, Ohio, United States \\ ${ }^{c}$ KBRwyle Aerospace Group, Ridgecrest, California, United States \\ ${ }^{\mathrm{d}}$ University of Central Florida, CREOL, Orlando, Florida, United States
}

\begin{abstract}
Fe:ZnSe and other transition metals have broad upper and lower energy manifolds that give rise to broad absorption and fluorescence bands in the mid-infrared spectral region. Energy transfer between $\mathrm{Fe}^{2+}$ ions via re-absorption of fluorescence (due to the spectral overlap of these bands) and phonon-assisted energy transfer (due to the lattice dynamics of $\mathrm{ZnSe}$ ) becomes more probable with increasing $\mathrm{Fe}^{2+}$ concentration. Here, we treat these processes as additional terms in the rate equations that govern the spontaneous decay of the $\mathrm{Fe}^{2+}$ ion. This treatment gives insight into anomalous behavior seen in the thermal lifetime of the Fe:ZnSe system. We also apply the model to other transition metal-doped II-VI materials. (c) The Authors. Published by SPIE under a Creative Commons Attribution 4.0 Unported License. Distribution or reproduction of this work in whole or in part requires full attribution of the original publication, including its DOI. [DOI: 10.1117/1.OE.60.5.056103]
\end{abstract}

Keywords: mid-infrared lasers; transition metals; vibronic lasers; spectroscopy; rate equations.

Paper 20201398 received Nov. 30, 2020; accepted for publication Apr. 21, 2021; published online May 19, 2021.

\section{Introduction}

In the past decade, Fe:ZnSe has developed into an important mid-infrared (IR) laser gain medium with rapid and substantial power scaling in both pulsed and continuous-wave regimes. ${ }^{1-9}$ Several studies have reported the temperature-dependent fluorescent lifetime of this material from 10 to $300 \mathrm{~K}$. However, the data produced by such studies differ substantially, ${ }^{1,10,11}$ and all studies report results that do not agree with the standard Mott-Seitz model ${ }^{12}$ of temperature-dependent fluorescent decay. In this work, which we initially presented in the conference proceedings of SPIE LASE, ${ }^{13}$ we develop a quantitative model that explains and resolves these discrepancies.

Vibronic laser gain media such as Fe:ZnSe and other transition metals have broad upper and lower energy manifolds that give rise to broad absorption and fluorescence bands. Energy transfer between active ions via re-absorption of fluorescence (due to the spectral overlap of these bands) and phonon-assisted energy transfer (due to the lattice dynamics of the crystal host) becomes more probable with increasing impurity concentration. We report a detailed model of the population dynamics of such systems, which includes contributions from fluorescent reabsorption, nonradiative energy transfer (NRET), and cascaded multi-phonon decay effects.

Spectroscopic measurements of the lifetime of several transition metal systems (including Fe:ZnSe) are presented and analyzed using this model. The model is fit to this lifetime data with excellent confidence intervals. The model shows qualitative agreement with expected trends, such as an increase in re-absorption and NRET with increasing ion concentration. The model provides additional insight for the design of mid-IR lasers based on Fe:ZnSe and other vibronic systems including systems based on $\mathrm{Cr}^{2+}$ and $\mathrm{Co}^{2+}$ ions.

*Address all correspondence to Jonathan W. Evans, jonathan.evans.6@us.af.mil 


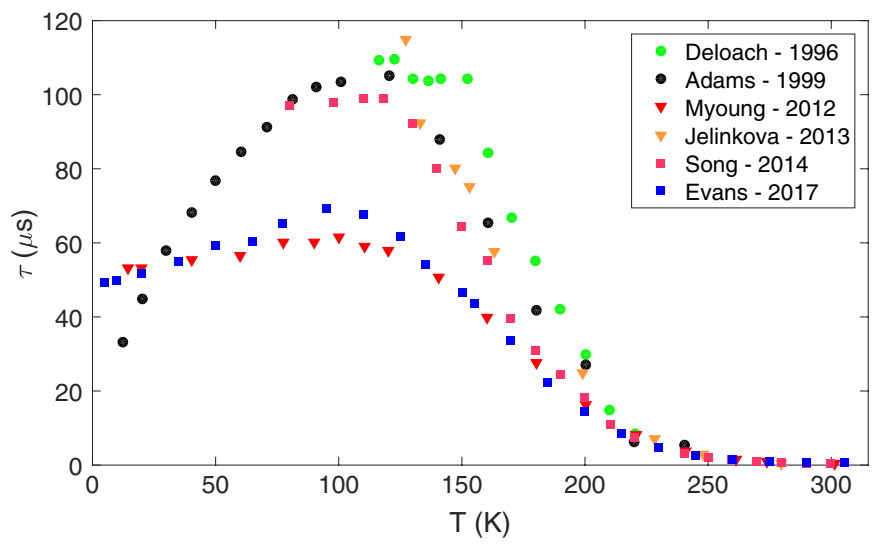

Fig. 1 The measured values of the fluorescence lifetime of $\mathrm{Fe}^{2+}$ ions in $\mathrm{ZnSe}$ with respect to changes in temperature.

\section{Background}

Many authors have measured the temperature-dependent fluorescent lifetime of Fe:ZnSe. Adams et al. ${ }^{14}$ and Song et al. ${ }^{15}$ have measured and reported the fluorescence lifetime of Fe:ZnSe with $\mathrm{Fe}^{2+}$ impurity concentrations $>10^{19}$ ions $/ \mathrm{cm}^{3}$. Their results show the lifetime of Fe:ZnSe to approach $105 \mu$ s near $120 \mathrm{~K}$. Deloach et al. ${ }^{16}$ and Jelínková et al. ${ }^{17}$ report similar results, though their concentrations were not specified. The data produced by these studies are shown in Fig. 1.

Myoung et al. ${ }^{18}$ measured the maximum value of the fluorescence lifetime of Fe:ZnSe with much lower $\mathrm{Fe}^{2+}$ concentrations of $10^{17}$ ions $/ \mathrm{cm}^{3}$ to be $\sim 60 \mu \mathrm{s}$ near $100 \mathrm{~K}$. In our recent work, ${ }^{11}$ we measured the maximum value of the fluorescence lifetime of Fe:ZnSe with intermediate $\mathrm{Fe}^{2+}$ concentrations of $10^{18}$ ions $/ \mathrm{cm}^{3}$ to be almost $70 \mu \mathrm{s}$ at $100 \mathrm{~K}$. These data are also included in Fig. 1.

It has been suggested that the reduction in the fluorescence lifetime observed by Myoung and in our work is attributable to the fact that the samples under investigation were produced with the post-growth thermal diffusion (PGTD) technique. However, the samples used by Song et al. ${ }^{15}$ were also produced in this manner, so that technique is apparently not the cause of the reduction in lifetime. We concluded in our recent work ${ }^{11}$ that the differences in the observed fluorescence lifetime from dataset to dataset are due to concentration effects. In that work, we developed a model of the population dynamics of such systems that includes contributions from effects that are known to be concentration dependent, namely, the re-absorption of fluorescence, NRET, and cascaded multi-phonon decay effects. In this work, we apply this model to additional lifetime data for Fe:ZnSe at different concentrations and Fe:CdMnTe, Cr:ZnS, Cr:ZnSe, Cr:CdSe, Cr:CdMnTe, Co:ZnS, Co:ZnSe, Co:ZnTe, and Co:CdTe.

\section{Theory}

In this section, we will develop a description of the fluorescent decay from the upper state manifold in which the low-energy population experiences NRET between impurity ions and the higher-energy population experiences significant re-absorption (with subsequent re-emission) of fluorescent radiation. NRET will shorten the observed lifetime from its intrinsic value and re-absorption will lengthen it (Henderson and Imbusch ${ }^{19}$ provide a broad overview of the electronic dynamics of vibronic systems at high impurity concentrations. They note that phononassisted NRET between active ions occurs with greater probability with decreasing temperature and increasing impurity concentration. They also note that re-absorption of fluorescent radiation occurs with greater probability with increasing spectral overlap and with increasing ion concentration. The spectral overlap of the absorption and emission bands of Fe:ZnSe increases with increasing temperature due to the broadening of those bands as more levels become thermally accessible.). The relative probabilities of these processes are mediated by the occupation statistics of the sublevels of the manifold, which are assumed to be in thermal equilibrium. 
We note that, in Fe:ZnSe, the energy difference between energy levels in the ${ }^{5} T_{2}$ manifold is much greater than $k_{b} T$, and thus thermal statistics cannot be neglected. Furthermore, $\mathrm{Fe}^{2+}$ ion concentration appears to play a significant role in determining the observed fluorescence lifetime of Fe:ZnSe. Consequently, we have developed a model that describes the temperature dependence of the fluorescence lifetime in terms of thermally activated and concentration-dependent decay processes.

\subsection{Rate Equation Modeling of Energy Transfer Mechanisms}

\subsubsection{Re-absorption}

Fe:ZnSe and other transition metal media operate as quasi-four level laser systems. However, for the sake of simplicity, we will use a two level approximation to the upper state manifold and a single level approximation to the lower state manifold. We have introduced an additional upper state level to describe some recycling of the upper manifold population due to re-absorption of fluorescent emission and subsequent re-emission. Figure 2 shows the level scheme used in the following discussion. We assume some initial population of the meta-level and ignore the initial population of the ground level.

In this model, the two upper levels (1) and (2) together represent a single meta-level and each experiences spontaneous relaxation with the same spontaneous decay rate $A_{20}=A_{10}$. With some probability $P_{r}$, a photon emitted from this meta-level is re-absorbed. This process is represented by $A_{12}$ and $A_{21}$. The probability of re-absorption (we will assume $100 \%$ re-emission of absorbed photons) is

$$
\begin{gathered}
P_{r}=\frac{A_{21}}{A_{21}+A_{20}}=A_{21} \tau, \\
=\frac{A_{12}}{A_{12}+A_{10}}=A_{12} \tau .
\end{gathered}
$$

So, $A_{21}=P_{r} / \tau$ where $\tau$ is the observed lifetime of the upper energy levels. It is worth noting that the re-absorption does not compete with spontaneous emission process, rather re-absorption of a photon occurs only after spontaneous emission of a photon. Thus, in this model of the movement of population due to re-absorption, the observed decay rate of the upper levels is $1 / \tau=A_{20}+A_{21}$. Consequently, $A_{20}=1 / \tau-A_{21}$.

We can write the rate equations for this system using the usual conventions:

$$
\begin{aligned}
\frac{d N_{2}}{d t} & =-A_{20} N_{2}-A_{21} N_{2}+A_{12} N_{1} \\
\frac{d N_{1}}{d t} & =-A_{10} N_{1}+A_{21} N_{2}-A_{12} N_{1} \\
\frac{d N_{0}}{d t} & =A_{20} N_{2}+A_{10} N_{1} .
\end{aligned}
$$

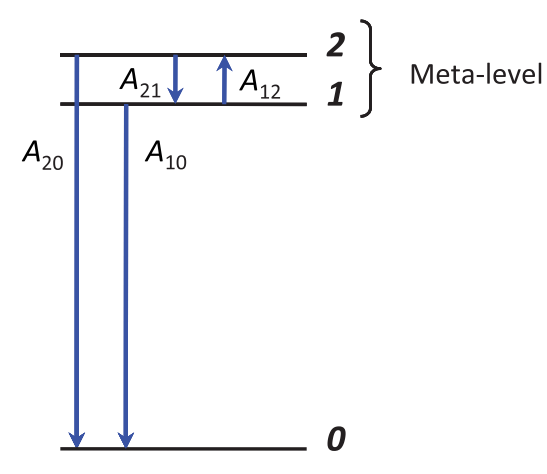

Fig. 2 An energy level diagram with levels (1) and (2) representing a single level with re-absorption. 


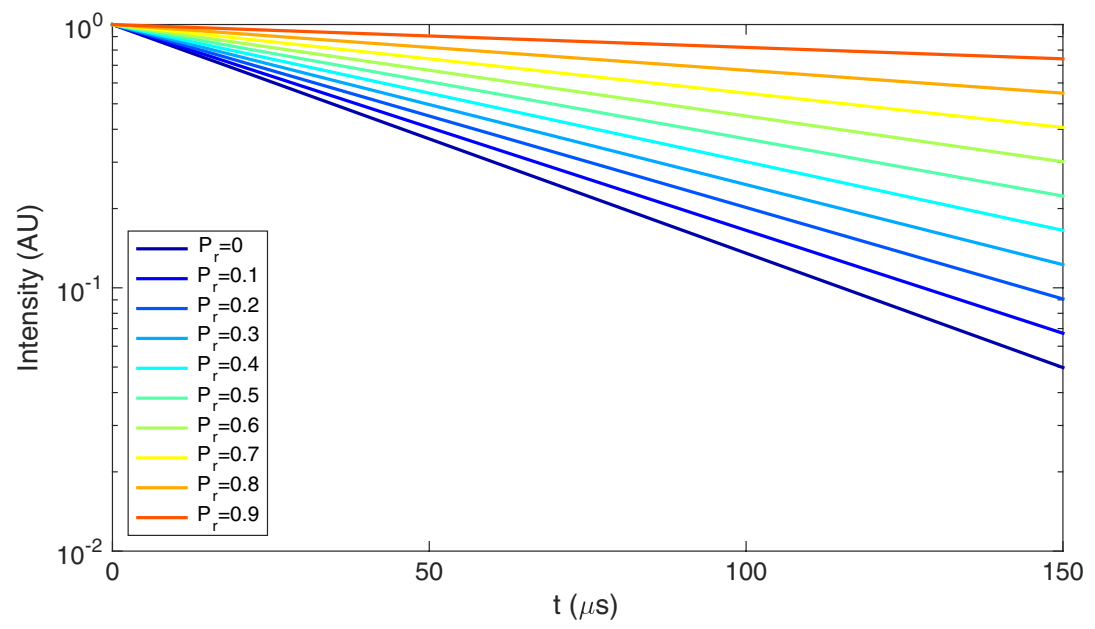

Fig. 3 Normalized traces of $I(t)$ with several values of the re-absorption probability $P_{r}$. The $P_{r}=0$ trace represents the spontaneous rate (In Figs. 3 and 5, we have used $\tau=50 \mu$ s for illustrative purposes.).

The fluorescence signal will be proportional to $I(t)=\left(1-P_{r}\right) N_{1}(t)+\left(1-P_{r}\right) N_{2}(t)$. Solving the system analytically, and substituting $A_{12} \rightarrow A_{21}, A_{10} \rightarrow A_{20}$, and $A_{20}=1 / \tau-A_{21}$, we can then substitute into the expression for $I(t)$ to obtain

$$
\begin{aligned}
I(t) & =e^{-t / \tau}\left(\left(N_{1}[0]+N_{2}[0]\right)\left(P_{r}-1\right) e^{P_{r} t / \tau}\right) \\
& =\left(N_{1}[0]+N_{2}[0]\right)\left(1-P_{r}\right) e^{-\left(1-P_{r}\right) t / \tau}
\end{aligned}
$$

where $N_{1}[0]$ and $N_{2}[0]$ are the initial population in levels (1) and (2), respectively. Figure 3 shows normalized traces of $I(t)$ as $P_{r}$ increases. It is worth noting that the apparent lifetime of the trace increases with increasing $P_{r}$.

\subsubsection{Non-radiative energy transfer}

It is straightforward to modify the rate equations to recast level (2) as a single upper level within the upper manifold and change level (1) to represent a radiation trap from which all paths to level (0) are nonradiative. Figure 4 shows the level scheme used in the following discussion.

We modify the previous system of rate equations using $A_{20}=1 / \tau$ and $A_{12}=A_{10}=0$ to represent non-radiative trapping, which occurs with some probability $P_{n}$. The solution for the fluorescence intensity becomes:

$$
\begin{aligned}
I(t) & =e^{-\left(1+P_{n}\right) t / \tau}\left(N_{2}[0]-N_{2}[0] P_{n}\right) \\
& =N_{2}[0]\left(1-P_{n}\right) e^{-\left(1+P_{n}\right) t / \tau} .
\end{aligned}
$$

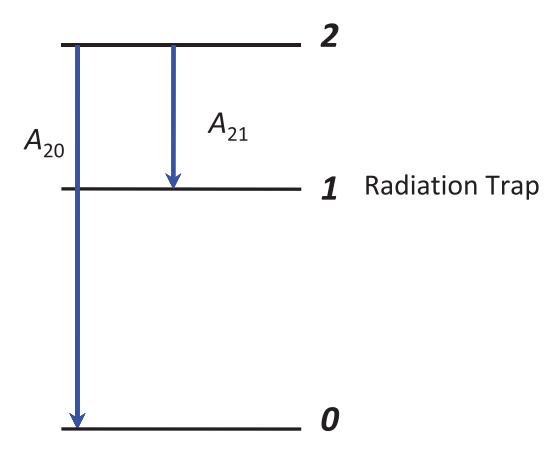

Fig. 4 Non-radiative energy transfer to a radiation trap. 
Evans et al.: Re-absorption and nonradiative energy transfer in vibronic laser gain media

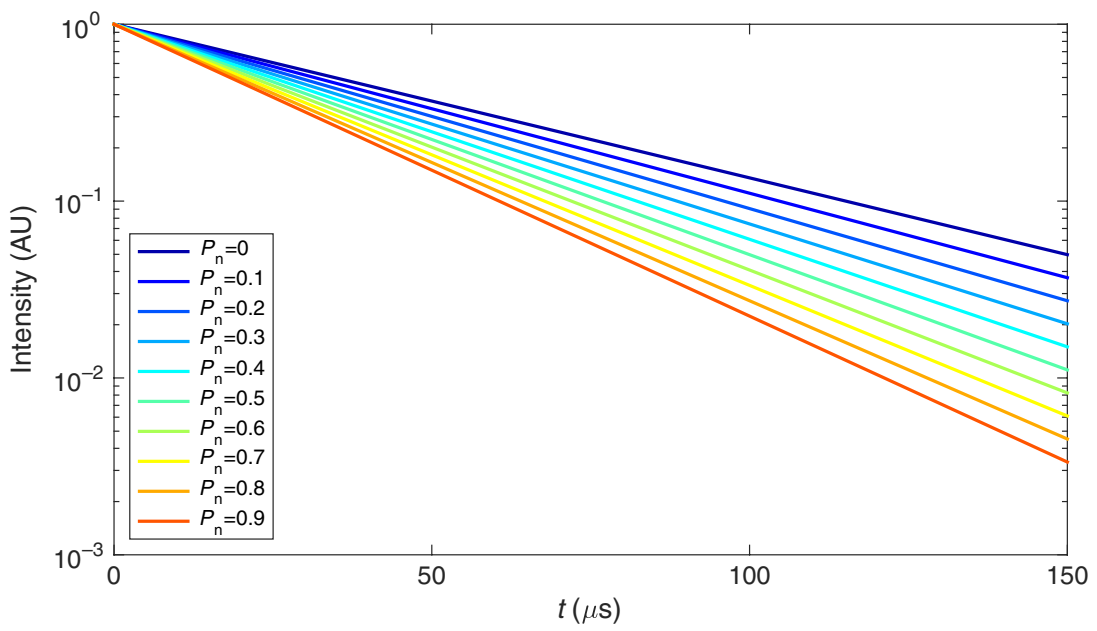

Fig. 5 Traces of $I(t)$ with several values of the re-absorption probability $P_{n}$.

Figure 5 shows normalized traces of $I(t)$ as $P_{n}$ increases. It is worth noting that the apparent lifetime of the decay decreases with increasing $P_{n}$.

\subsubsection{Combining re-absorption and NRET}

Modeling the combined system requires the introduction of two additional energy levels as shown in Fig. 6. The system of rate equations becomes slightly more complicated but can still be solved analytically. The model takes into consideration the florescence contribution from two separate levels, one of which sees the re-absorption of fluorescence and the other of which experiences radiation trapping. In this configuration, we suppose them to be separated by some activation energy, which is a free parameter in a previous model. Figure 6 shows this system.

The rate equations can be written as

$$
\begin{aligned}
\frac{d N_{4}}{d t} & =-A_{40} N_{4}-A_{43} N_{4}+A_{34} N_{3} \\
\frac{d N_{3}}{d t} & =-A_{30} N_{3}-A_{34} N_{3}+A_{43} N_{4} \\
\frac{d N_{2}}{d t} & =-A_{20} N_{2}-A_{21} N_{2} \\
\frac{d N_{1}}{d t} & =A_{21} N_{2} \\
\frac{d N_{0}}{d t} & =A_{40} N_{4}+A_{30} N_{3}+A_{20} N_{2} .
\end{aligned}
$$

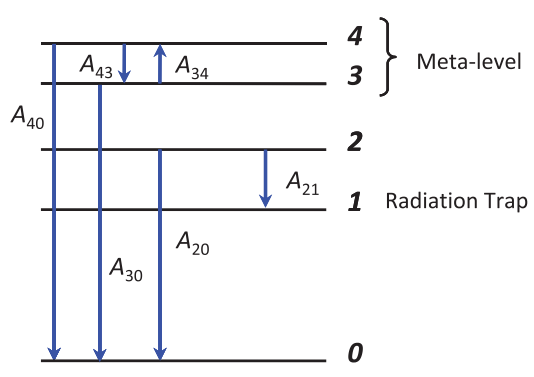

Fig. 6 An electronic level diagram with levels (3) and (4) representing a single level with reabsorption and level (1) representing a radiation trap. Levels (1)-(4) belong to the upper state manifold. 


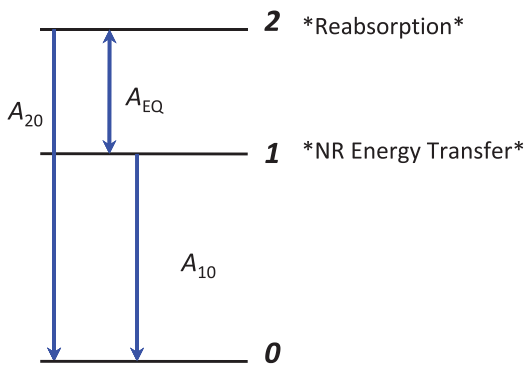

Fig. 7 Two levels, which undergo different parasitic processes, in thermal equilibrium.

The fluorescence signal is

$$
I(t) \propto\left(1-P_{r}\right)\left(N_{4}[t]+N_{3}[t]\right)+\left(1-P_{n}\right) N_{2}[t],
$$

which gives

$$
\begin{aligned}
I(t)= & -e^{-\left(2+P_{n}+P_{r}\right) t / \tau} \cdot\left(N_{2}[0] e^{\left(1+P_{r}\right) t / \tau}\left(P_{n}-1\right)\right. \\
& \left.+\left(N_{3}[0]+N_{4}[0]\right) e^{\left(1+P_{n}+2 P_{r}\right) t / \tau}\left(P_{r}-1\right)\right) \\
= & N_{2}[0]\left(1-P_{n}\right) e^{-\left(1+P_{n}\right) t / \tau} \\
& +\left(N_{3}[0]+N_{4}[0]\right)\left(1-P_{r}\right) e^{-\left(1-P_{r}\right) t / \tau}
\end{aligned}
$$

Clearly, the solution to this system represents decay from two independent levels with different rates to the same ground level. So, we simplify the five-level to its three-level equivalent (see Fig. 7). In this system, level (2) experiences a different relaxation rate than level (1) because photons emitted spontaneously from level (2) are re-absorbed with probability $P_{r}$ and some of the population of level (1) moves to the ground state non-radiatively with probability $P_{n}$.

Now, suppose that the system obeys Fermi-Dirac statistics and is initially at equilibrium. So,

$$
\begin{aligned}
& N_{1}[0]=N_{\text {total }} \cdot \kappa_{1}=N_{\text {total }}\left(1+\frac{G_{2}}{G_{1}} \exp \left(\frac{-\Delta E}{k_{b} T}\right)\right)^{-1}, \\
& N_{2}[0]=N_{\text {total }} \cdot \kappa_{2}=N_{\text {total }}\left(1+\frac{G_{1}}{G_{2}} \exp \left(\frac{\Delta E}{k_{b} T}\right)\right)^{-1},
\end{aligned}
$$

where $\Delta E=E_{2}-E_{1}$ is the energy difference between levels (1) and (2).

However, since the decay rates are different, the system is not in equilibrium for $t>0$ unless $P_{r}=P_{n}=0$, which gives the uninteresting solution: $I(t)=N_{\text {total }} e^{-t / \tau}$. So, we force the system to maintain thermal equilibrium by adding the equilibrium term to the system of rate equations (Fig. 7). A term $A_{E Q}$ represents the movement of the population to maintain thermal equilibrium. It is worth noting that the re-thermalization of the excited state manifold should happen on time scales much faster than the spontaneous lifetimes of any of the levels.

The rate equations are straightforward:

$$
\begin{aligned}
& \frac{d N_{2}}{d t}=-A_{20} N_{2}-\left(N_{2}-N_{1}\right) A_{E Q} \\
& \frac{d N_{1}}{d t}=-A_{10} N_{1}+\left(N_{2}-N_{1}\right) A_{E Q} \\
& \frac{d N_{0}}{d t}=A_{20} N_{2}+A_{10} N_{1} .
\end{aligned}
$$

We impose the condition:

$$
\frac{N_{2}[t]}{N_{1}[t]}=\frac{N_{2}[0]}{N_{1}[0]}
$$


and solve for $A_{E Q}$ to obtain:

$$
A_{E Q}=\frac{N_{2}[t] N_{1}[t]}{N_{1}^{2}[t]-N_{2}^{2}[t]} \frac{\tau_{1}-\tau_{2}}{\tau_{1} \tau_{2}}
$$

where $\tau_{1}=1 / A_{10}$, and $\tau_{2}=1 / A_{20}$.

Substituting this expression back into Eq. (11) and solving again provides a new expression for the decay of the upper manifold. Thus,

$$
I(t) \propto\left(1-P_{r}\right) N_{2}[t]+\left(1-P_{n}\right) N_{1}[t],
$$

becomes

$$
\begin{aligned}
I(t) & =\left(N_{2}[0]\left(1-P_{r}\right)+N_{1}[0]\left(1-P_{n}\right)\right) \exp \left(-\frac{N_{2}[0] t \tau_{1}+N_{1}[0] t \tau_{2}}{N_{2}[0] \tau_{1} \tau_{2}+N_{1}[0] \tau_{1} \tau_{2}}\right) \\
& =K \exp \left(\frac{-N_{2}[0] t}{\left(N_{2}[0]+N_{1}[0]\right) \tau_{2}}\right) \exp \left(\frac{-N_{1}[0] t}{\left(N_{2}[0]+N_{1}[0]\right) \tau_{1}}\right) \\
& =K \exp \left(\frac{-\kappa_{2} t}{\left(\kappa_{2}+\kappa_{1}\right) \tau_{2}}\right) \exp \left(\frac{-\kappa_{1} t}{\left(\kappa_{2}+\kappa_{1}\right) \tau_{1}}\right),
\end{aligned}
$$

where $K=N_{2}[0]\left(1-P_{r}\right)+N_{1}[0]\left(1-P_{n}\right)$. It is worth noting that

$$
\kappa_{2}+\kappa_{1}=\left(1+\frac{G_{1}}{G_{2}} \exp \left(\frac{\Delta E}{k_{b} T}\right)\right)^{-1}>+\left(1+\frac{G_{2}}{G_{1}} \exp \left(-\frac{\Delta E}{k_{b} T}\right)\right)^{-1}=1
$$

So,

$$
I(t)=K \exp \left(-\frac{\kappa_{2} t}{\tau_{2}}\right) \exp \left(-\frac{\kappa_{1} t}{\tau_{1}}\right)
$$

The product of exponentials is an exponential, so the form of the spontaneous decay of the manifold is clearly a single exponential with a lifetime $\tau$, and

$$
\frac{1}{\tau}=\frac{\kappa_{1}}{\tau_{1}}+\frac{\kappa_{2}}{\tau_{2}} .
$$

Using $W=1 / \tau$, and substituting explicitly for $\kappa_{2}$ and $\kappa_{1}$, we obtain:

$$
W_{\mathrm{obs}}=\frac{W_{1}}{1+\frac{G_{2}}{G_{1}} \exp \left(-\frac{\Delta E}{k_{b} T}\right)}+\frac{W_{2}}{1+\frac{G_{1}}{G_{2}} \exp \left(\frac{\Delta E}{k_{b} T}\right)} .
$$

\subsection{A Shorter Derivation}

In this section, we offer a shorter derivation of Eq. (19) without the need for full rate equation analysis. Neglecting, for now, the energy distribution of its vibronic sublevels, the average relaxation rate of the upper manifold is simply the weighted sum of the relaxation rates of each of its electronic levels

$$
N_{\text {total }} W_{\text {obs }}=\sum_{n=1}^{M} N_{n} W_{n},
$$

where the total population is $N_{\text {total }}=\sum_{n} N_{n}$ and $M$ is the total number of sublevels. For simplicity, we treat the manifold as consisting of two energy nondegenerate meta-levels. Level (1) has a population $N_{1}$ and undergoes NRET with some probability $P_{1}$. Level (2) has a population $N_{2}$ and undergoes re-absorption with some probability $P_{2}$ (we assume $100 \%$ re-emission). 
It is useful to define a parameter that describes the partitioning of the manifold into these two meta-levels. The ratio of the degeneracies $G_{1}$ and $G_{2}$ of the meta-levels is

$$
\frac{G_{2}}{G_{1}}=\frac{\sum_{n=m+1}^{M} g_{n}}{\sum_{n=1}^{m} g_{n}},
$$

where $M$ is the total number of sublevels in the manifold and $g_{n}$ is the degeneracy factor of the $n^{\text {th }}$ level. However, this partitioning of the manifold is arbitrary, so $G_{2} / G_{1}$ must be treated as a fit parameter.

We introduce thermal statistics by noting that the fractional population distribution is described as

$$
\frac{N_{2}}{N_{1}}=\frac{G_{2}}{G_{1}} \exp \left(-\frac{\Delta E}{k_{b} T}\right)
$$

where $\Delta E$ is the separation of the two meta-levels. Substituting Eq. (22) into Eq. (20), we find

$$
W_{\mathrm{obs}}=\frac{W_{1}}{1+\frac{G_{2}}{G_{1}} \exp \left(-\frac{\Delta E}{k_{b} T}\right)}+\frac{W_{2}}{1+\frac{G_{1}}{G_{2}} \exp \left(\frac{\Delta E}{k_{b} T}\right)}
$$

\subsection{Multi-Phonon Cascade}

The Mott-Seitz model describes the effect of temperature on the fluorescence lifetime of active ions decaying to the ground state by spontaneous emission in competition with a non-radiative process such as multi-phonon cascade (MPC). ${ }^{12,19,20}$ The measured lifetime $\tau_{\text {obs }}$ of the decay depends on the thermally dependent probability of the non-radiative decay process. The observed lifetime is

$$
\tau_{\mathrm{obs}}=\tau_{\mathrm{rad}} \cdot \eta=\tau_{\mathrm{rad}} \cdot \frac{A_{r}}{A_{r}+A_{n r} \exp \left(-E / k_{b} T\right)},
$$

where $\tau_{\text {rad }}$ is the lifetime of the ion due to spontaneous radiative decay only, $\eta$ is the fluorescence efficiency of the aggregate decay process, $A_{r}$ is the probability of radiative decay, $A_{n r}$ is the probability of nonradiative decay, and the exponential term is the familiar Boltzmann statistical term with an activation energy $E$. This expression can be rewritten as

$$
\tau_{\mathrm{obs}}=\frac{\tau_{\mathrm{rad}}}{1+C \exp \left(-E / k_{b} T\right)},
$$

where $C$ describes the magnitude of nonradiative decay. We recast Eq. (25) in terms of related rates:

$$
W_{\text {obs }}=W_{\text {rad }}\left(1+C \exp \left(-E / k_{b} T\right)\right)=W_{\text {rad }}+C^{\prime} \exp \left(-E / k_{b} T\right)=W_{\text {rad }}+W_{n r} .
$$

We use this functional form as the basis for the construction of this model. We will define the total decay rate $W_{\text {total }}=W_{\text {obs }}+W_{n r}$, where $W_{n r}$ is a nonradiative decay term that we will introduce below and $W_{\mathrm{obs}}$ is the internal rate we derived in the previous sections:

$$
W_{\mathrm{obs}}=\frac{W_{1}}{1+\frac{G_{2}}{G_{1}} \exp \left(-\frac{\Delta E}{k_{b} T}\right)}+\frac{W_{2}}{1+\frac{G_{1}}{G_{2}} \exp \left(\frac{\Delta E}{k_{b} T}\right)} .
$$

NRET will increase the decay rate $W_{1}$ relative to the spontaneous rate. In the absence of NRET, $W_{1}$ will approach the radiative rate. Similarly, RET will artificially lengthen the observed fluorescence lifetime; this effect is equivalent to a decrease in the decay rate $W_{2}$ relative to the spontaneous rate. In the absence of RET, $W_{2}$ will approach the radiative rate. In the absence of 
both effects, $W_{\text {rad }}$ will approach the radiative rate at all temperatures. Thus, we see that this model is merely a more general form of the Mott-Seitz model.

We now replace the nonradiative term $W_{n r}$ in Eq. (26) with the Miyakawa-Dexter expression for nonradiative quenching (NRQ) due to $\mathrm{MPC}^{21}$ to obtain

$$
W_{\text {total }}=W_{\mathrm{obs}}+W_{M P C}^{(p)}
$$

where

$$
W_{M P C}^{(p)}=\frac{A}{M^{2} \sqrt{2 \pi p}}\left(\frac{S e}{p}\right)^{p-2}\left(1+\frac{1}{\exp \left(\frac{h \nu_{e f f}}{k_{b} T}\right)-1}\right)^{p}
$$

and where $k_{b}$ is the Boltzmann's constant, $e$ is the Euler's constant, $A$ is a coupling constant for the nonradiative term, $M$ is an effective mass, $S$ is the Huang-Rhys parameter, $T$ is the temperature, and $h \nu_{\text {eff }}=E / p$ is the effective phonon energy required for $p$ phonons to bridge the energy gap $E$ between the energy levels involved in the optical transition.

Using this approach, we can construct a model for the observed fluorescence lifetime $\tau_{\text {obs }}=1 / W_{\text {total }}$ that includes both energy transfer and MPC:

$$
\begin{aligned}
W_{\text {total }}= & \frac{W_{1}}{1+\frac{G_{2}}{G_{1}} \exp \left(-\frac{\Delta E}{k_{b} T}\right)}+\frac{W_{2}}{1+\frac{G_{1}}{G_{2}} \exp \left(\frac{\Delta E}{k_{b} T}\right)} \\
& +\frac{A}{M^{2} \sqrt{2 \pi p}}\left(\frac{S e}{p}\right)^{p-2}\left(1+\frac{1}{\exp \left(\frac{h \nu_{\text {eff }}}{k_{b} T}\right)-1}\right)^{p} .
\end{aligned}
$$

\section{Measuring the Fluorescence Lifetime}

We performed measurements of the fluorescence lifetime of $\mathrm{Fe}^{2+}$ ions in chemical vapor deposition-grown ZnSe using a periodically poled lithium niobate (PPLN) optical parametric oscillator (OPO) pumped by a Spectra-Physics $\mathrm{Nd}: \mathrm{YVO}_{4}$ laser. The pump laser operated at $1 \mathrm{kHz}$ and the PPLN grating had a quasi-phasemathing period of $31 \mu \mathrm{m}$. Thus, at $100^{\circ} \mathrm{C}$, the OPO emitted at $3050 \mathrm{~nm}$. The full-width-half-maximum linewidth of the output was $8 \mathrm{~nm}$. The average power was $>20 \mathrm{~mW}$ and the average pulse length was $\sim 30 \mathrm{~ns}$. Pulses from the OPO were focused into a sample of Fe:ZnSe, which was wrapped in indium foil and pressure mounted to a cold-finger within an evacuated cryostat. The decay of the optical output was recorded by focusing fluorescent emission through an Acton Spectra-Pro 750 monochromator onto a liquid-nitrogen cooled InSb detector.

The fluorescent decay signals were recorded with an oscilloscope and the lifetime $\tau$ of the impurity ions was determined as a least-sum-squared error fit of

$$
I(t)=A \exp \left(-\frac{t-t_{0}}{\tau}\right)+a_{0}
$$

to the measured decay trace of the collected fluorescence. Figure 8 shows a typical fit for an Fe:ZnSe sample.

We performed measurements of the fluorescence lifetime of other transition metal gain media using the same pulsed excitation technique. Short laser pulses were focused into the sample of interest, which was wrapped in indium foil and pressure mounted to a cooling mount. Different pump lasers were used for the various media because no single pump laser was appropriate for all samples. The decay of the optical output was recorded by focusing fluorescent emission onto an appropriate photodiode and Eq. (31) was fit to the decay trace to determine the lifetime.

In the sections that follow, we will fit Eq. (30) to the datasets we have collected in this way as well as to datasets we have sampled from the relevant literature. Because we have access to the raw decay traces from our dataset, we are confident that these data are well fit by a single 


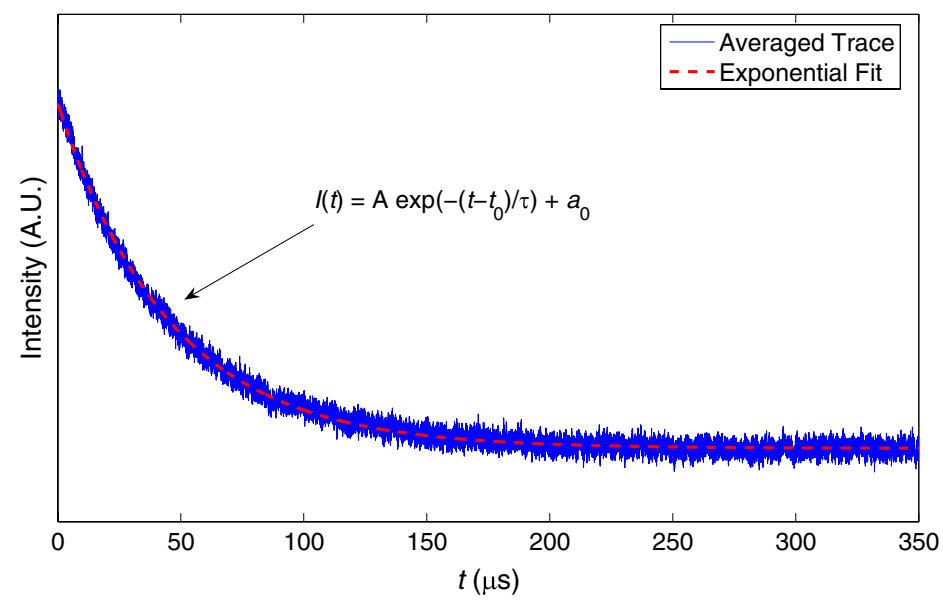

Fig. 8 A numerical fit of Eq. (31) to an experimental decay trace from Fe:ZnSe.

exponential decay model characterized by a single florescent lifetime $\tau$. As we have shown, this single-exponential behavior is to be expected from a multiplicity of single-exponential processes that proceed simultaneously in a region that is (nearly) in thermal equilibrium. We do not have access to the decay traces for the literature data to which we have also fit our model. In some cases, the multi-variable fit has a lower degree of confidence than we obtained for fits to our dataset. This can be partially explained by three considerations: First, our data were taken using a great degree of computer control, which was not available to some earlier researchers. Second, the literate data were sampled graphically from published figures, which will introduce some degree of discretization error. Third, the energy transfer processes in the materials that other researchers have analyzed may not proceed in equilibrium, in which cases the observed decay may not be well characterized by a single exponential. Nevertheless, a single exponential decay model has been used in the lifetimes reported in all cases. Despite these sources of potential error, we show that Eq. (30) accommodates trends in temperature that are a general feature of such datasets.

\section{Analysis}

We recast $\nu_{\text {eff }}$ in Eq. (30) in terms of $p$ by substituting $h \nu_{\text {eff }}=\Delta / p$, where $\Delta=10 D q$ is the crystal field energy of the impurity ion and the value of $\Delta$ is obtained spectroscopically. So, we obtain a model of the form

$$
\begin{aligned}
W_{\text {total }}= & \frac{W_{1}}{1+\frac{G_{2}}{G_{1}} \exp \left(-\frac{\Delta E}{k_{b} T}\right)}+\frac{W_{2}}{1+\frac{G_{1}}{G_{2}} \exp \left(\frac{\Delta E}{k_{b} T}\right)} \\
& +\frac{A}{M^{2} \sqrt{2 \pi p}}\left(\frac{S e}{p}\right)^{p-2}\left(1+\frac{1}{\exp \left(\frac{10 D q / p}{k_{b} T}\right)-1}\right)^{p},
\end{aligned}
$$

which includes seven fit parameters: $\tau_{1}=1 / W_{1}, \tau_{2}=1 / W_{2}, G_{2} / G_{1}, \Delta E, A / M^{2}, S$, and $p$. The model can then be fit to the decay traces of various transition metal laser media.

\section{1 $\mathrm{Fe}^{2+}$-doped Materials}

In our previous work, ${ }^{11}$ we fit Eq. (32) to the data we collected at that time and to two other measurements of the thermally resolved lifetime of Fe:ZnSe from the literature. The results are discussed in detail in that work; we summarize those results here briefly. Equation (32) fits the data very well and the confidence intervals are small relative to the fluorescence lifetime. Figures 9(a)-9(c) shows the confidence intervals of the numerical fit are reasonable. 
Evans et al.: Re-absorption and nonradiative energy transfer in vibronic laser gain media

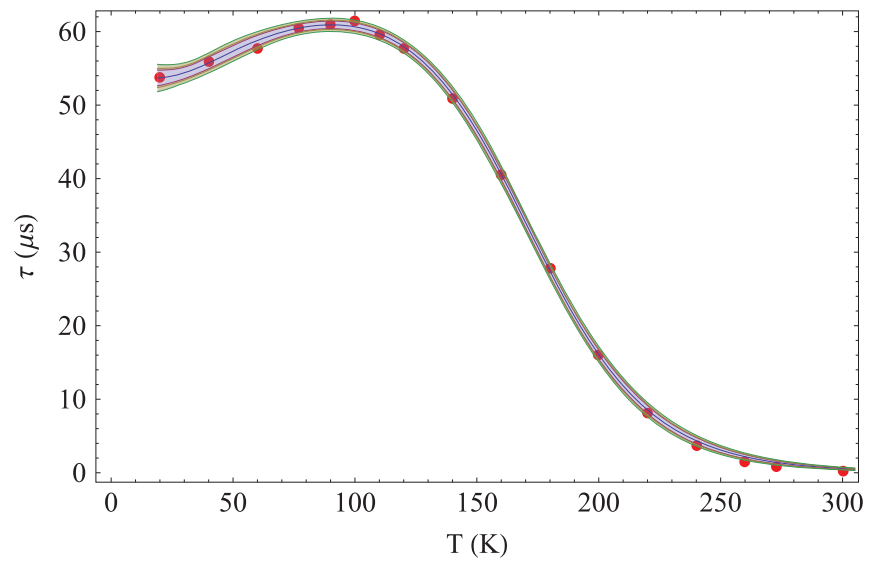

Myoung et al.

(a)

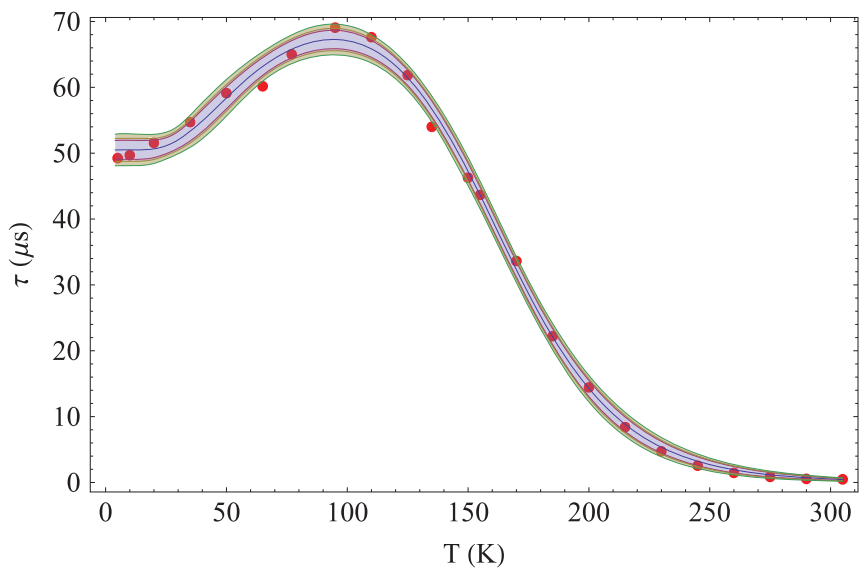

Evans et al.

(b)

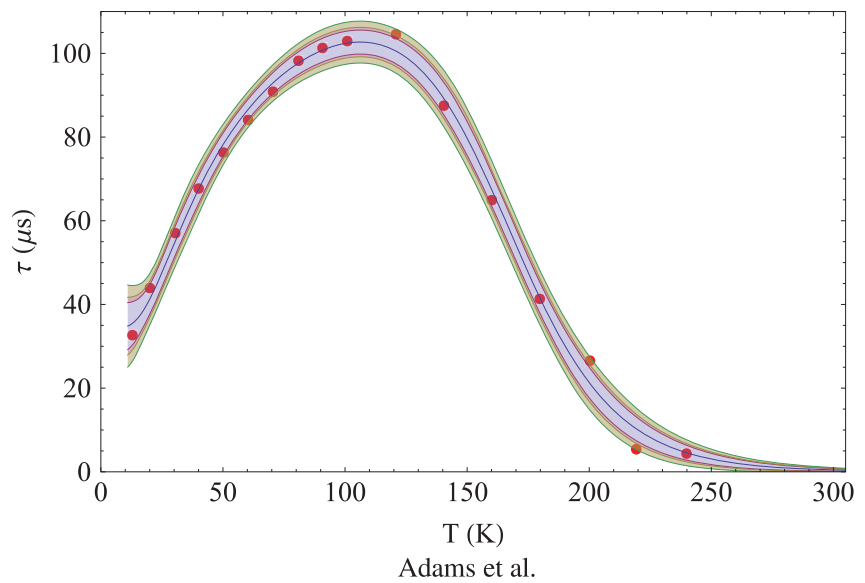

(c)

Fig. 9 A numerical fit of Eq. (30) to the lifetime data for Fe:ZnSe from the (a) Myoung et al., ${ }^{18}$ (b) Evans et al., ${ }^{11}$ and (c) Adams et al. ${ }^{14}$ Colored bands show the $90 \%, 95 \%$, and $99 \%$ confidence intervals.

The fit parameters of Eq. (32) are shown in Table 1. The parameters $A / M^{2}, S$, and $p$, which are associated with nonradiative decay via MPC, are similar across all samples. The remaining parameters are associated with the radiative decay process and with energy transfer are seen to vary across samples. We hypothesized that concentration effects are responsible for the differences observed in these parameters between samples. 
Table 1 The returned values of the fit parameters for our model of the temperature-dependent fluorescence lifetime of $\mathrm{Fe}^{2+}$ ions in $\mathrm{ZnSe}$. It is worthy to note that $A / M^{2}, p$, and $S$ are similar across columns.

\begin{tabular}{lccc}
\hline \hline & Myoung $^{18}$ & Evans $^{11}$ & Adams $^{14}$ \\
\hline $\mathrm{N}\left(\mathrm{cm}^{-3}\right)$ & $1.0 \times 10^{17}$ & $8.6 \times 10^{18}$ & $\sim 5 \times 10^{19}$ \\
$\tau_{1}(\mu \mathrm{s})$ & $54.5 \pm 0.6$ & $51.8 \pm 1.0$ & $34.5 \pm 3.6$ \\
$\tau_{2}(\mu \mathrm{s})$ & $\sim 80$ & $\sim 108$ & $\sim 245$ \\
$G_{2} / G_{1}$ & $4.84 \pm 0.25$ & $5.64 \pm 1.8$ & $7.5 \pm 0.9$ \\
$\Delta E\left(\mathrm{~cm}^{-1}\right)$ & $90.1 \pm 15.9$ & $94.0 \pm 16.0$ & $47.8 \pm 5.9$ \\
$A / M^{2}\left(\mathrm{~ms}^{-1}\right)$ & $10.8 \pm 0.7$ & $10.2 \pm 1.2$ & $9.8 \pm 2.4$ \\
$S$ & 5.46 & 5.47 & 5.44 \\
$p$ & $16.0 \pm 0.7$ & $15.69 \pm 0.3$ & $17.2 \pm 0.8$ \\
\hline \hline
\end{tabular}

\subsubsection{Fe:ZnSe}

In this work, we repeated the measurements of our previous work. ${ }^{11}$ We automated our data collection using LabVIEW and used a more stable laser (described in the experimental section) with a higher pulse repetition frequency for improved smoothing via averaging. The signal-tonoise ratio improvement achieved using this technique is evident in the confidence intervals of parameters of the fits. We examined Fe:ZnSe samples with various concentrations to eliminate experimental variables from the conclusion that concentration is the key variable affecting the lifetime of Fe:ZnSe. Each of the samples was purchased from IPG Photonics and the concentrations quoted by the vendor span two orders of magnitude. The fit of Eq. (32) to each of the samples is shown in Figs. 10(a)-10(c).

A notable trend with respect to concentration is observed. With increasing concentration, the curvature of lifetime curve with respect to temperature increases significantly with increasing $\mathrm{Fe}^{2+}$ concentration.

For comparison, a sample of Fe:ZnSe was grown solvothermally. Interestingly, the behavior of that material is consistent with the Mott-Seitz model (see Fig. 11). One might assume that a sample would approach that regime in the limit of low concentration, but the concentration of this sample, as inferred from optical transmission $N=-\log \left(I / I_{0}\right) / \sigma L$, is about the same as the sample interrogated to produce Fig. 10(b). Clearly the trends differ significantly, so concentration is not the only variable contributing to these observations.

The two samples are fabricated by different growth methods; the IPG crystal was made via PGTD and the in-house crystal by solvothermal growth. This is worth noting because previous work has shown that Fe:ZnSe lasers based on crystals grown by PGTD behave significantly different after treatment under hot isostatic press (HIP), ${ }^{22}$ which suggests that crystals grown via PGTD are highly defective. Such defects may increase the probability of inter-ionic energy transfer. Thus, it is possible that the observed differences in the thermal lifetime are due to energy transfer processes coupled to these defects.

Analysis of the data in this section shows that measured value of the lifetime below $260 \mathrm{~K}$ depends, in part, on the volume concentration of the $\mathrm{Fe}^{2+}$ dopant. Above $260 \mathrm{~K}$, however, the effect of concentration is seen to be less significant. As shown in Fig. 12, the measured value of the lifetime from many samples of different ionic concentrations converge into relative agreement at temperatures $>260 \mathrm{~K}$. It is worth noting that the literature values were determined graphically, and some degree of sampling error is present in both Fig. 12 and Table 2.

The data from Fig. 12 near room temperature are shown in Table 2. Also included is a room temperature measurement of the fluorescent lifetime of $\mathrm{Fe}^{2+}$ ions in $\mathrm{ZnSe}$ nanoparticles reported by Martinez. ${ }^{23}$ The measured value of the fluorescent lifetime is approximately $500 \mathrm{~ns}$ across all concentrations. Values between 300 and $400 \mathrm{~ns}$ are reported at the highest and lowest values of concentration. No clear trend of the measured lifetime with respect to concentration can be discerned. This topic should be further investigated. 
Evans et al.: Re-absorption and nonradiative energy transfer in vibronic laser gain media

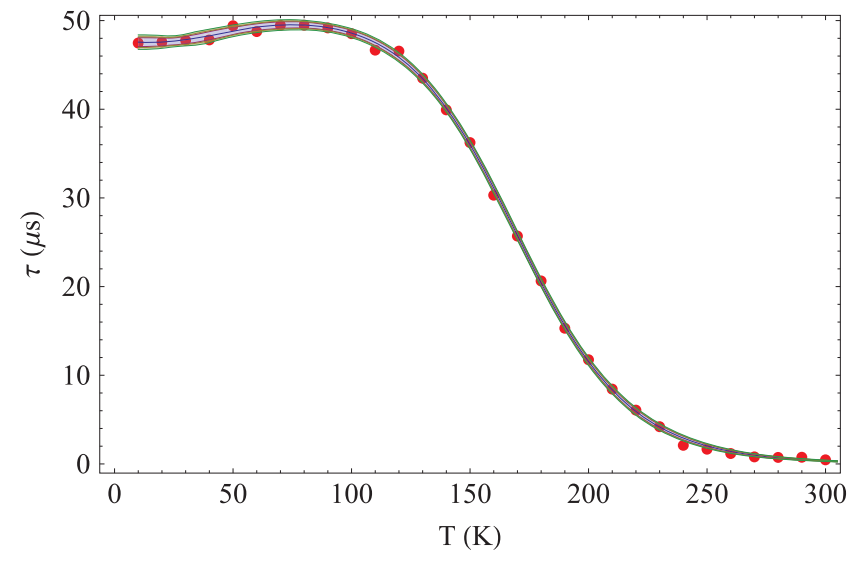

$N=8.45 \times 10^{17} \mathrm{~cm}^{-3}$

(a)

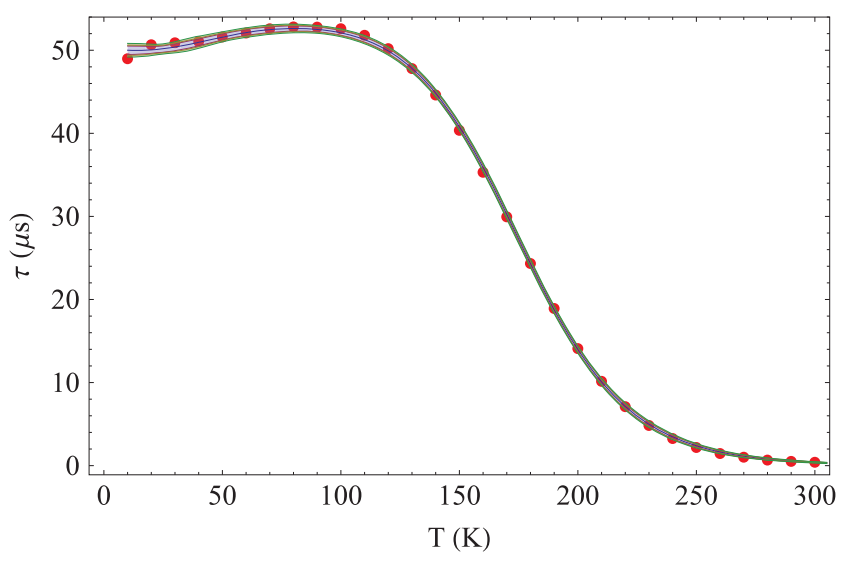

$N=4.8 \times 10^{18} \mathrm{~cm}^{-3}$

(b)

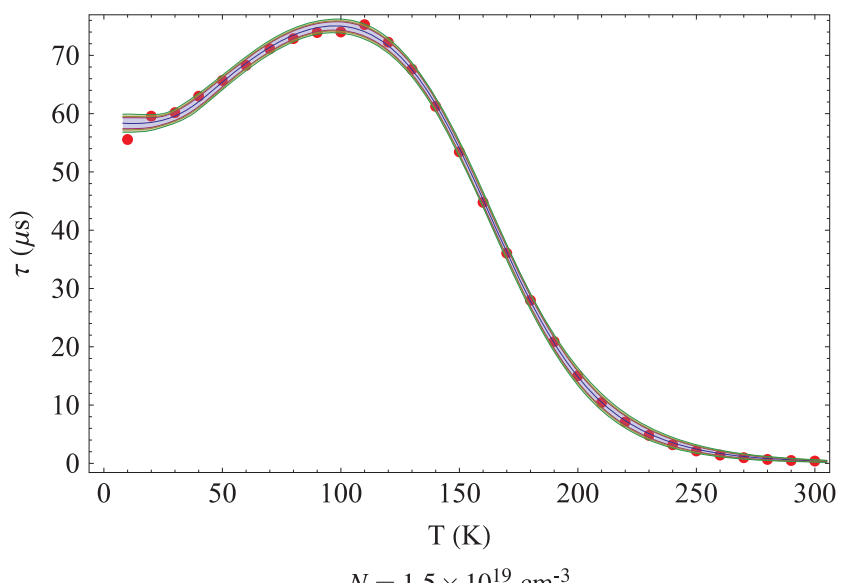

(c)

Fig. 10 A numerical fit of Eq. (32) to the lifetime data for diffusion-doped Fe:ZnSe from IPG: (a) sample 1, (b) sample 2, and (c) sample 3.

\subsubsection{Fe:CdMnTe}

A sample of $\mathrm{Fe}: \mathrm{Cd}_{\mathrm{x}} \mathrm{Mn}_{1-\mathrm{x}} \mathrm{Te}$ was cut from a boule grown from melt by Brimrose Corporation using the Bridgman technique with an $\mathrm{Fe}^{2+}$ impurity concentration of $2.7 \times 10^{18}$ ions $/ \mathrm{cm}^{3}$ and an alloying fraction of approximately $x=0.9$. Mid-IR fluorescence was generated in the sample via excitation with $\sim 40$-ns laser pulses centered at a wavelength of $3.45 \mu \mathrm{m}$ from MgO:PPLN 
Evans et al.: Re-absorption and nonradiative energy transfer in vibronic laser gain media

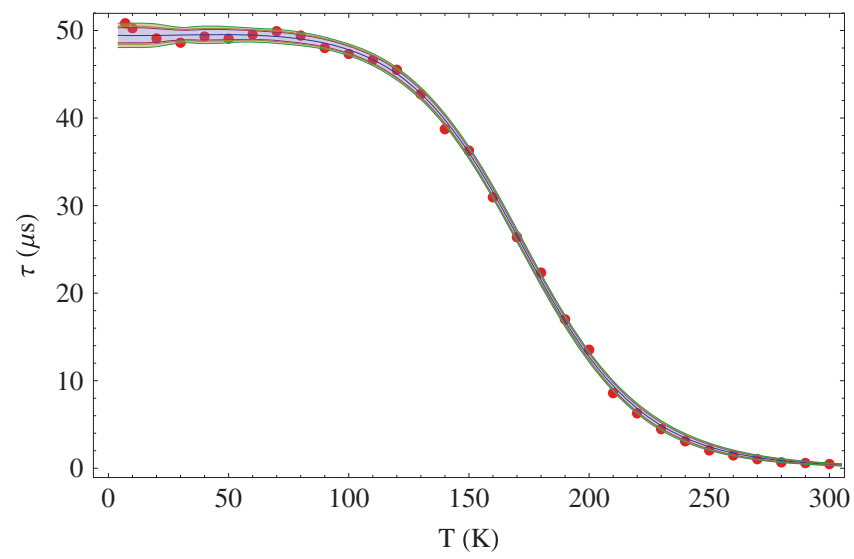

Fig. 11 A numerical fit of Eq. (32) to the lifetime data for solvothermally grown Fe:ZnSe $\left(N \approx 5.7 \times 10^{18} \mathrm{~cm}^{-3}\right)$.

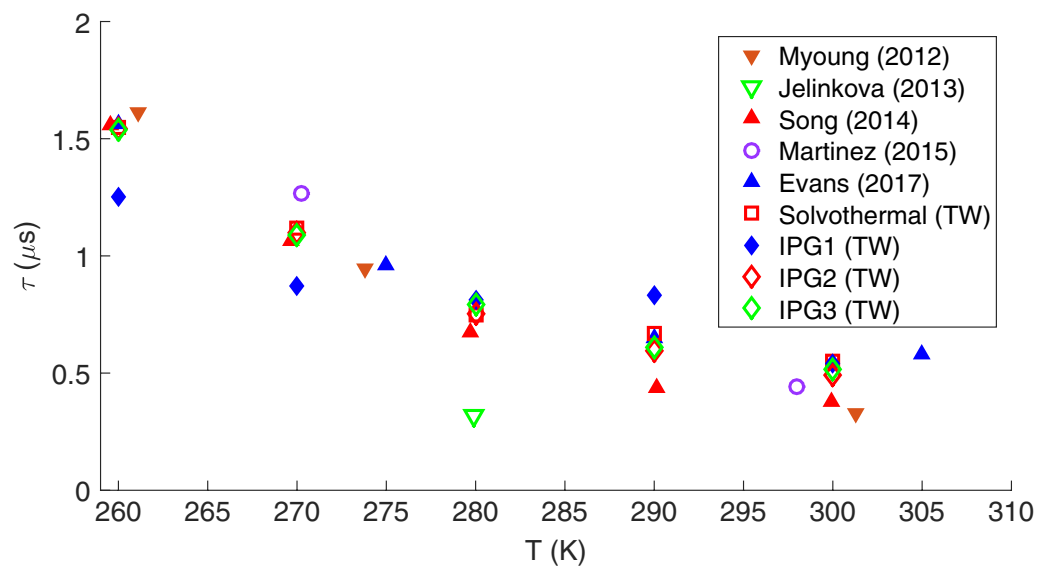

Fig. 12 The measured values of the fluorescence lifetime of $\mathrm{Fe}^{2+}$ ions in $\mathrm{ZnSe}$ as reported in literature and as measured in this work (TW).

Table 2 The measured values of the fluorescence lifetime of $\mathrm{Fe}^{2+}$ ions in $\mathrm{ZnSe}$ at room temperature as reported in literature and as measured in this work.

\begin{tabular}{lccc}
\hline \hline Source & $\mathrm{T}(\mathrm{K})$ & $\mathrm{N}\left(\mathrm{cm}^{-3}\right)$ & $\tau(\mathrm{ns})$ \\
\hline Myoung et al. $^{18}$ & 301 & $1 \times 10^{17}$ & 326.8 \\
Song et al. $^{15}$ & 300 & $1.5 \times 10^{19}$ & 377.1 \\
Martinez $^{23}$ & 299 & - & 444.1 \\
Evans $^{11}$ & 305 & $8.6 \times 10^{18}$ & 581.7 \\
Solvothermal (TW) $^{18}$ & 300 & $5.7 \times 10^{18}$ & 550.8 \\
IPG 1 (TW) $^{\text {IPG 2 (TW) }}$ & 300 & $8.45 \times 10^{17}$ & 541.6 \\
IPG 3 (TW) & 300 & $4.8 \times 10^{18}$ & 489.7 \\
\hline \hline
\end{tabular}




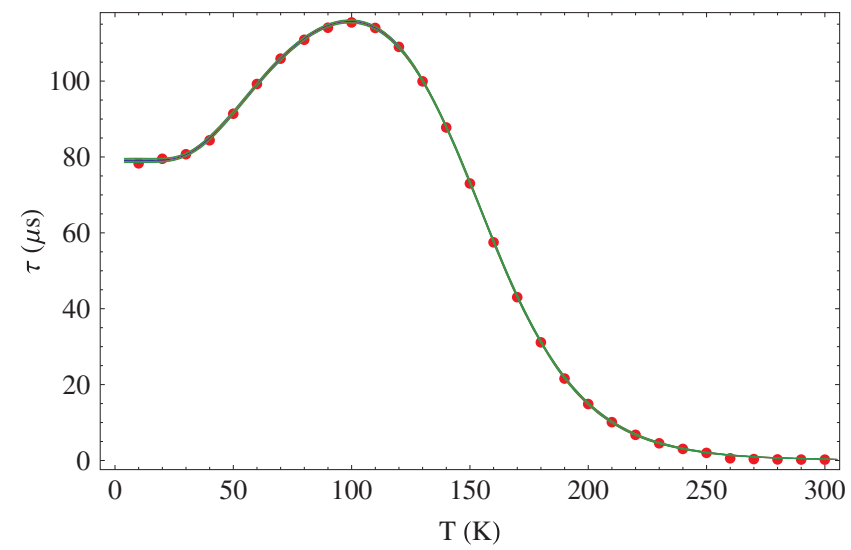

Fig. 13 A numerical fit of Eq. (32) to the lifetime data for Fe:CdMnTe $\left(N \approx 2.7 \times 10^{18} \mathrm{~cm}^{-3}\right)$. It is worthy to note the incredibly tight confidence intervals of the fit.

Table 3 The returned values of the fit parameters for our model of the temperature-dependent fluorescence lifetime of $\mathrm{Fe}^{2+}$ ions in $\mathrm{ZnSe}$. It is worthy to note that $p$ and $S$ are similar across the Fe:ZnSe samples.

\begin{tabular}{lccccc}
\hline \hline & Fe:ZnSe & Fe:ZnSe & Fe:ZnSe & Fe:ZnSe & \\
\cline { 2 - 4 } & IPG sample 1 & IPG sample 2 & IPG sample 3 & Solvothermal & Fe:CdMnTe \\
\hline $\mathrm{N}\left(\mathrm{cm}^{-3}\right)$ & $8.5 \times 10^{17}$ & $4.8 \times 10^{18}$ & $1.5 \times 10^{19}$ & $\sim 5.7 \times 10^{18}$ & $2.7 \times 10^{18}$ \\
$\tau_{1}(\mu \mathrm{s})$ & $48.3 \pm 0.3$ & $50.5 \pm 0.3$ & $59.3 \pm 0.6$ & $50.3 \pm 0.5$ & $80.0 \pm .2$ \\
$\tau_{2}(\mu \mathrm{s})$ & $54.1 \pm 5.7$ & $58.3 \pm 7.5$ & $\sim 122$ & $\sim 52$ & $195 \pm 12.9$ \\
$G_{2} / G_{1}$ & 6.54 & 4.30 & 4.44 & 4.94 & $9.0 \pm 1.6$ \\
$\Delta E\left(\mathrm{~cm}^{-1}\right)$ & $101 \pm 68.6$ & $90.0 \pm 50.8$ & $94.0 \pm 16.0$ & $\sim 110$ & $116 \pm 4.7$ \\
$A / M^{2}\left(\mathrm{~ms}^{-1}\right)$ & $18.6 \pm 0.9$ & $12.3 \pm 0.5$ & $10.4 \pm 1.0$ & $10.4 \pm 0.5$ & $27.2 \pm 0.3$ \\
$S$ & 5.35 & 5.44 & 5.50 & 5.49 & 5.84 \\
$p$ & $16.4 \pm 0.2$ & $16.7 \pm 0.2$ & $16.4 \pm 0.4$ & $16.1 \pm 0.4$ & $16.5 \pm 0.1$ \\
\hline \hline
\end{tabular}

OPO $(\Lambda=30.5 \mu \mathrm{m})$. The decay traces were recorded directly using an InSb detector and a digital oscilloscope using the same setup as the previous section. The temperature dependent fluorescence lifetimes and the fit of Eq. (32) to that data are shown in Fig. 13. The fit parameters are shown in Table 3.

\section{2 $\mathrm{Cr}^{2+}$-Doped Materials}

Thermally dependent lifetime datasets for $\mathrm{Cr}: \mathrm{ZnS}, \mathrm{Cr}: \mathrm{ZnSe}$, and Cr:ZnTe were obtained from DeLoach et al. ${ }^{16}$ and were fit using Eq. (32). Likewise, the thermally dependent lifetime datasets for $\mathrm{Cr}: \mathrm{ZnSe}$ and $\mathrm{Cr}: \mathrm{CdSe}$ were obtained from $\mathrm{McKay}^{24}$ and were fit to the same equation. The fit parameters for each material are shown in Table 4. The data and the corresponding fits are shown in Fig. 14 (Cr:ZnS), Fig. 15 (Cr:ZnTe), Figs. 16(a) and 16(b) (Cr:ZnSe), and Fig. 17 (Cr:CdSe).

\section{3 $\mathrm{Co}^{2+}$-Doped Materials}

Thermally dependent lifetime datasets for Co:ZnS, Co:ZnSe, and Co:ZnTe were obtained from DeLoach et al. ${ }^{16}$ and were fit using Eq. (32). The thermally dependent lifetime dataset for Co:CdTe collected for this work was also used to fit to the same equation. The fit parameters for each material are shown in Table 5. The data and the corresponding fits are shown in Fig. 18 (Co:ZnS), Fig. 19 (Co:ZnSe), Fig. 20 (Co:ZnTe), and Fig. 21 (Co:CdTe). 
Table 4 Fit parameters for the fluorescence lifetime several $\mathrm{Cr}^{2+}$-doped samples. The fit for $\mathrm{Cr}: \mathrm{ZnTe}$ is not consistent with the other materials and the data does not fit our model well. The data may indicate strong concentration quenching effects.

\begin{tabular}{lcccc}
\hline \hline & Cr:ZnS & Cr:ZnSe & Cr:ZnTe & Cr:CdSe \\
\cline { 2 - 5 } & DeLoach et al. $^{16}$ & McKay $^{24}$ & DeLoach et al. $^{16}$ & McKay $^{24}$ \\
\hline $\mathrm{N}\left(\mathrm{cm}^{-3}\right)$ & $1.8 \times 10^{19}$ & $1 \times 10^{18}$ & $5 \times 10^{18}$ & $(2-6) \times 10^{18}$ \\
$\tau_{1}(\mu \mathrm{s})$ & $6.81 \pm 0.07$ & $5.21 \pm 0.07$ & $2.63 \pm 0.14$ & $5.37 \pm 0.088$ \\
$\tau_{2}(\mu \mathrm{s})$ & $8.7 \pm 1.3$ & $12.5 \pm 0.6$ & 8.7 & $6.00 \pm 0.08$ \\
$G_{2} / G_{1}$ & 4.08 & 3.11 & 0.66 & 4 \\
$\Delta E\left(\mathrm{~cm}^{-1}\right)$ & $144 \pm 38$ & $188 \pm 14.0$ & 150 & 150 \\
$A / M^{2}\left(\mu \mathrm{s}^{-1}\right)$ & $1.006 \pm 0.0067$ & $0.433 \pm 0.024$ & 0.011 & $2.86 \pm 0.005$ \\
$S$ & $5.14 \pm 0.07$ & $5.11 \pm .06$ & 5.03 & 5.35 \\
$p$ & $27.3 \pm 0.6$ & $29.5 \pm 1.7$ & 15.8 & $25.0 \pm 0.7$ \\
\hline \hline
\end{tabular}

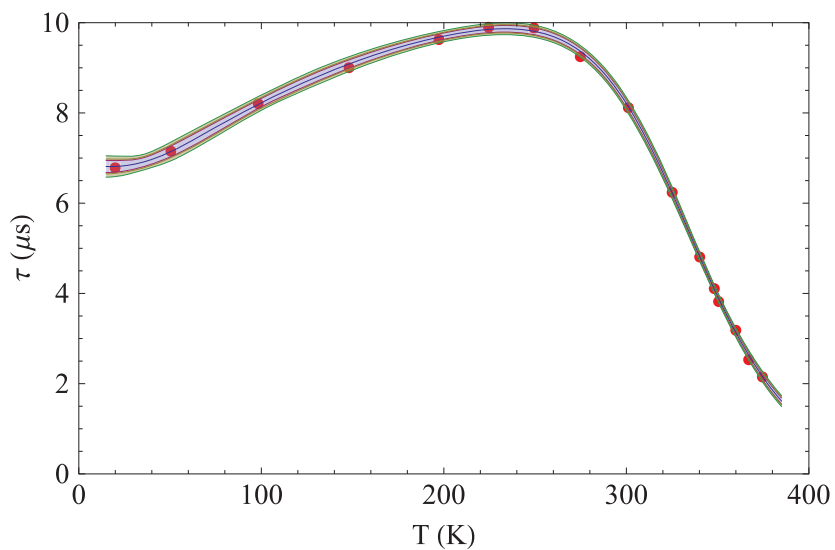

Fig. $14 \mathrm{~A}$ numerical fit of Eq. (32) to the lifetime data for Cr:ZnS from DeLoach et al. ${ }^{16}$ $\left(N \approx 1.8 \times 10^{19} \mathrm{~cm}^{-3}\right)$ Colored bands show the $90 \%, 95 \%$, and $99 \%$ confidence intervals.

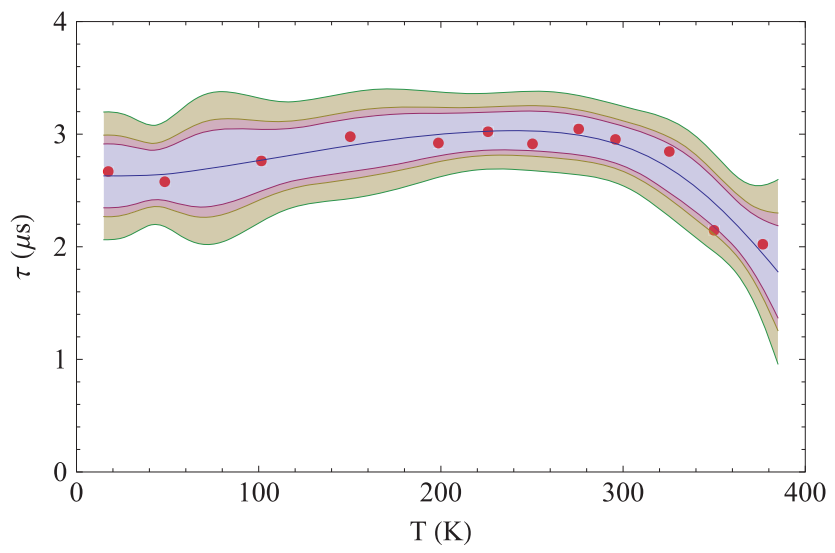

Fig. 15 A numerical fit of Eq. (32) to the lifetime data for Cr:ZnTe from DeLoach et al. ${ }^{16}$ $\left(N \approx 5 \times 10^{18} \mathrm{~cm}^{-3}\right)$ Colored bands show the $90 \%, 95 \%$, and $99 \%$ confidence intervals. 
Evans et al.: Re-absorption and nonradiative energy transfer in vibronic laser gain media

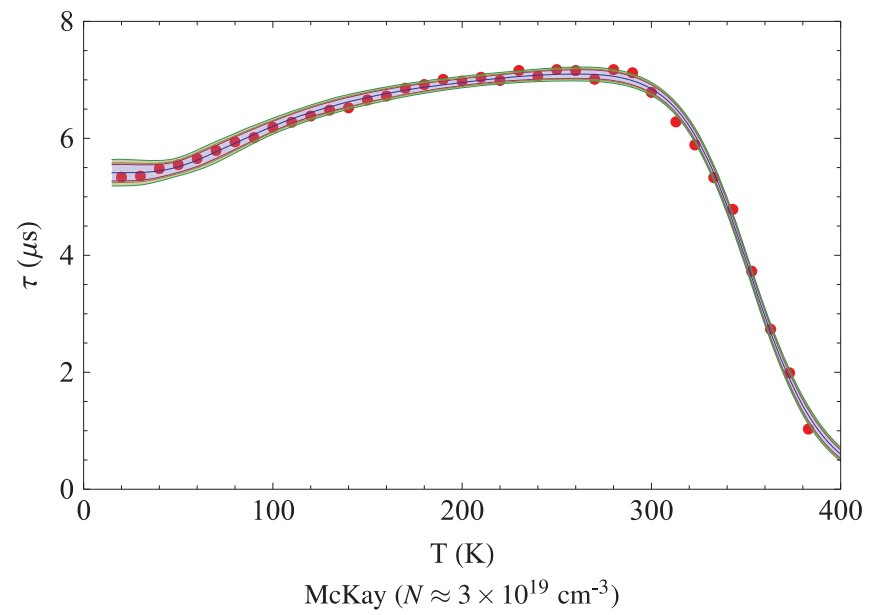

(a)

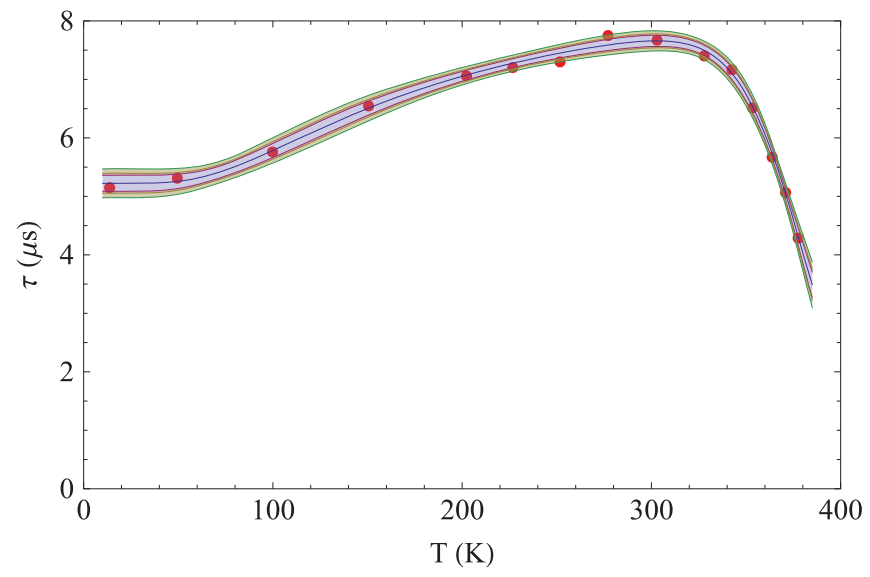

$\operatorname{DeLoach}\left(N \approx 1 \times 10^{18} \mathrm{~cm}^{-3}\right)$

(b)

Fig. 16 A numerical fit of Eq. (32) to the lifetime data for Cr:ZnSe from (a) McKay ${ }^{24}$ and (b) Deloach. ${ }^{16}$ Colored bands show the $90 \%, 95 \%$, and $99 \%$ confidence intervals.

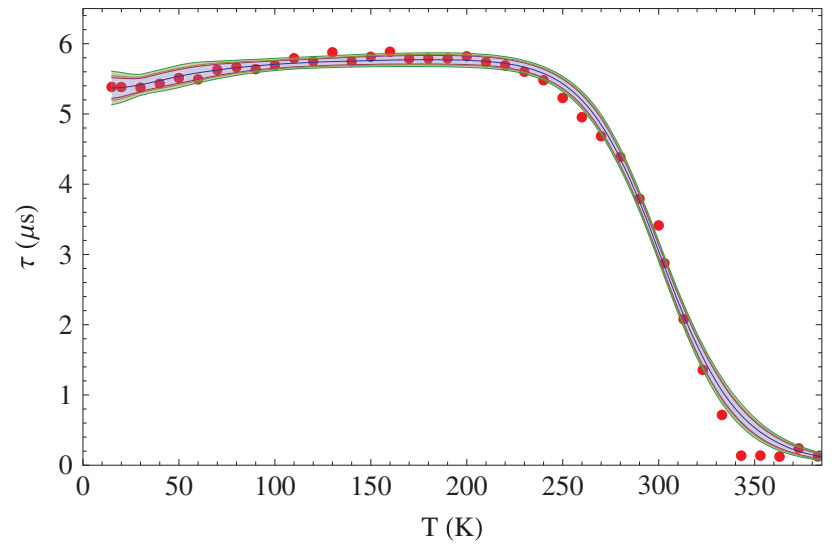

Fig. $17 \mathrm{~A}$ numerical fit of Eq. (32) to the lifetime data for Cr:CdSe from McKay ${ }^{24}$ $\left(N \approx 1.7-5.9 \times 10^{18} \mathrm{~cm}^{-3}\right)$. Colored bands show the $90 \%, 95 \%$, and $99 \%$ confidence intervals. 
Table 5 Fit parameters for the fluorescence lifetime several $\mathrm{Co}^{2+}$-doped samples. The fits for $\mathrm{Co}: \mathrm{ZnS}$ and $\mathrm{Co}: \mathrm{ZnTe}$ did not require an additional radiative term. It is worth noting the dramatic difference in $\tau_{1}$ from host to host. The fit for $\mathrm{Co}$ :CdTe did not depend critically on the value of $\tau_{2}$ and the values of $S$ and $p$ returned do not seem consistent with the other materials shown.

\begin{tabular}{lcccc}
\hline \hline & Co:ZnS & Co:ZnSe & Co:ZnTe & Co:CdTe \\
\cline { 2 - 5 } & DeLoach et al. ${ }^{16}$ & DeLoach et al. ${ }^{16}$ & DeLoach et al. ${ }^{16}$ & This work \\
\hline $\mathrm{N}\left(\mathrm{cm}^{-3}\right)$ & $1.8 \times 10^{19}$ & $3 \times 10^{18}$ & $2.5 \times 10^{19}$ & $3.5 \times 10^{19}$ \\
$\tau_{1}(\mu \mathrm{s})$ & $1180 \pm 6.8$ & 904 & $588.8 \pm 10.0$ & $591 \pm 3.6$ \\
$\tau_{2}(\mu \mathrm{s})$ & - & $1318 \pm 65$ & - & $>1000$ \\
$G_{2} / G_{1}$ & - & 4 & - & 4 \\
$\Delta E\left(\mathrm{~cm}^{-1}\right)$ & - & $202 \pm 19$ & - & $159.5 \pm 7.1$ \\
$A / M^{2}\left(\mathrm{~ms}^{-1}\right)$ & $0.467 \pm 0.040$ & $1.29 \pm 0.14$ & $0.33 \pm 0.072$ & $0.247 \pm 0.061$ \\
$S$ & 4.98 & 4.24 & 4.68 & $8.61 \pm .053$ \\
$p$ & $19.3 \pm 0.5$ & $22.2 \pm 0.6$ & $21.9 \pm 1.8$ & $32.7 \pm 0.73$ \\
\hline \hline
\end{tabular}

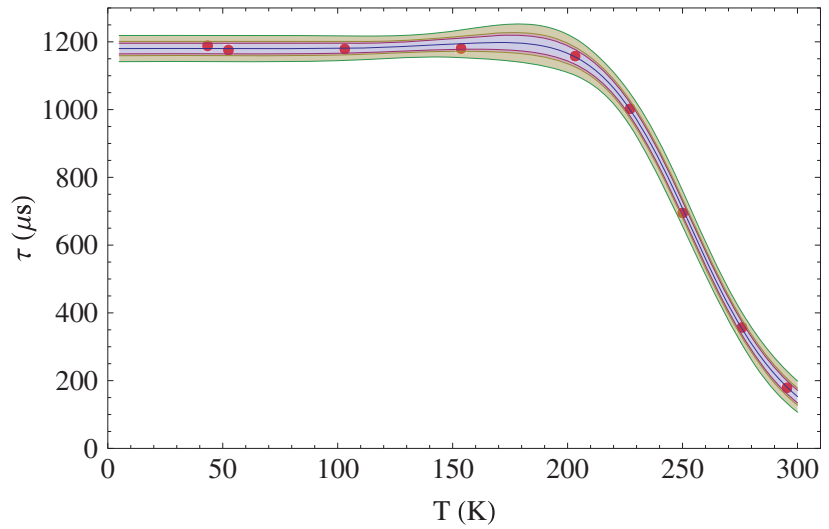

Fig. $18 \mathrm{~A}$ numerical fit of Eq. (32) to the lifetime data for Co:ZnS from DeLoach et al. ${ }^{16}$ $\left(N \approx 1.7 \times 10^{19} \mathrm{~cm}^{-3}\right)$ Colored bands show the $90 \%, 95 \%$, and $99 \%$ confidence intervals.

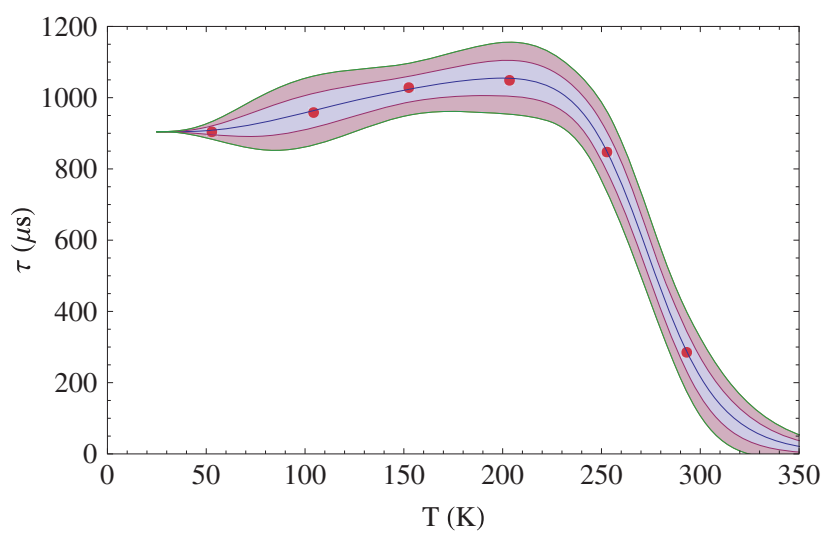

Fig. 19 A numerical fit of Eq. (32) to the lifetime data for Co:ZnSe from DeLoach et al. ${ }^{16}$ $\left(N \approx 3 \times 10^{18} \mathrm{~cm}^{-3}\right)$ Colored bands show the $90 \%$ and $95 \%$ confidence intervals. 
Evans et al.: Re-absorption and nonradiative energy transfer in vibronic laser gain media

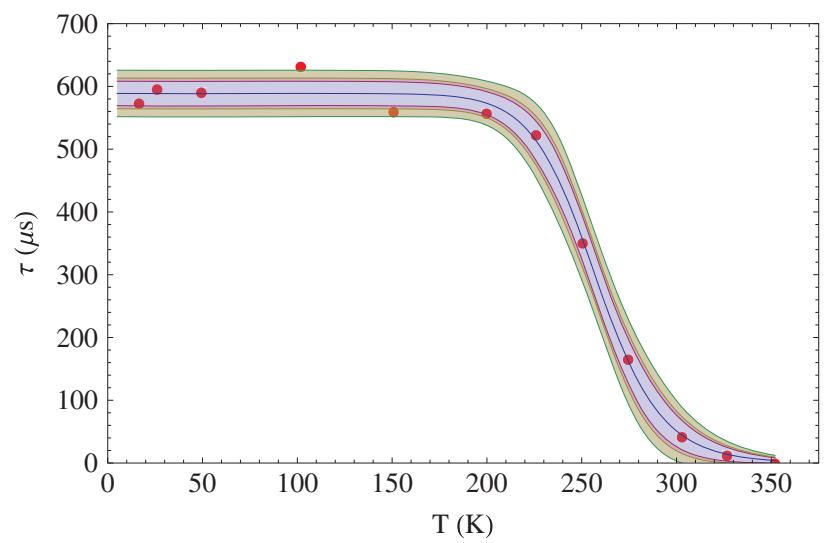

Fig. 20 A numerical fit of Eq. (32) to the lifetime data for Co:ZnTe from DeLoach et al. ${ }^{16}$ $\left(N \approx 2.5 \times 10^{19} \mathrm{~cm}^{-3}\right)$ Colored bands show the $90 \%, 95 \%$, and $99 \%$ confidence intervals.

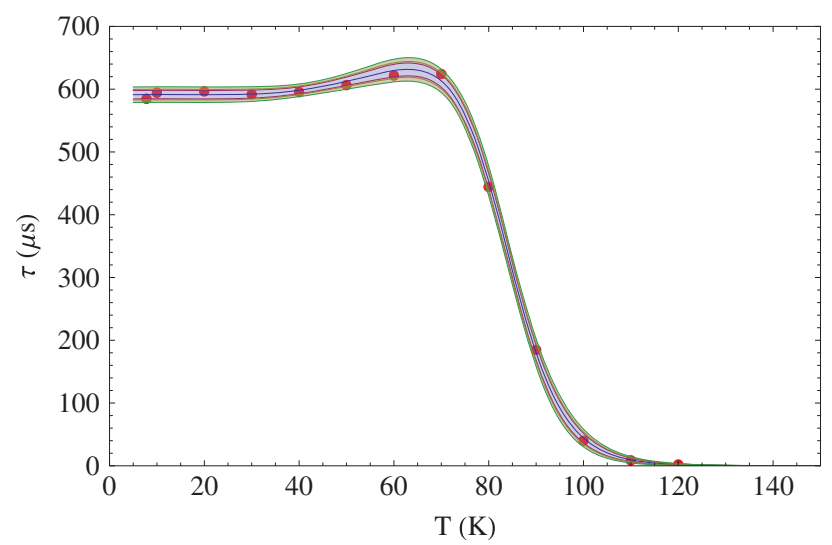

Fig. 21 A numerical fit of Eq. (32) to the lifetime data for Co:CdTe taken as a part of this work $\left(N \approx 2.5 \times 10^{19} \mathrm{~cm}^{-3}\right)$. Colored bands show the $90 \%, 95 \%$, and $99 \%$ confidence intervals.

\section{Tables}

This section presents values returned from the fit of Eq. (32):

$$
\begin{aligned}
W_{\text {total }}= & \frac{W_{1}}{1+\frac{G_{2}}{G_{1}} \exp \left(-\frac{\Delta E}{k_{b} T}\right)}+\frac{W_{2}}{1+\frac{G_{1}}{G_{2}} \exp \left(\frac{\Delta E}{k_{b} T}\right)} \\
& +\frac{A}{M^{2} \sqrt{2 \pi p}}\left(\frac{S e}{p}\right)^{p-2}\left(1+\frac{1}{\exp \left(\frac{10 D q / p}{k_{b} T}\right)-1}\right)^{p}
\end{aligned}
$$

to the data shown in the Analysis section. Values proceeded by a " " are approximate. Values in bold had to be held fixed for the fit to converge. Values reported without uncertainties are known to high degrees of confidence, so the uncertainties are omitted for formatting reasons.

\section{Conclusions}

The model we have detailed in this work fits several measurements of the thermally resolved fluorescent lifetime for several II-VI crystals with different ionic dopants and with various impurity concentrations. The model offers a quantitative explanation for observations in transition metal laser gain media that have not been previously explained. Our analysis of the data for 
Fe:ZnSe shows conclusively that the $\mathrm{Fe}^{2+}$ concentration of the samples drives departure from the Mott-Seitz model for thermally dependent fluorescent lifetime. The model also provides strong evidence that NRQ via MPC is responsible for the temperate-activated reduction of the fluorescence lifetime in several classes of transition metal II-VI materials.

One interesting implication of this work-especially for Fe:ZnSe lasers, for which the lifetime of the sample can be double its radiative value-is that the energy storage lifetime of the gain medium can be increased, without decreasing the cross-section of the transition, as is the case in crystal field engineering techniques. This is possible because the observed increase in lifetime is due to re-absorption effects, not to a reduction in the spontaneous decay probability of the optical transition. Consequently, the saturation intensity is not affected.

Due to the large amount of available data, the trends in Fe:ZnSe are well understood, but future work will be required to understand the trends in other materials. Future work will focus on collecting more lifetime data for materials, which are promising candidates for use in mid-IR lasers.

\section{Acknowledgments}

We acknowledge and thank the Air Force Office of Scientific Research (AFOSR) and the Sensors Directorate of the Air Force Research Laboratory (AFRL) for funding this research. We thank Glen Perram of the Air Force Institute of Technology for technical discussions. Additionally, we thank Pamela Evans for proofreading this manuscript.

\section{References}

1. J. J. Adams et al., "Tunable laser action at 4.0 microns from Fe:ZnSe," in Adv. Solid State Lasers, Optical Society of America, p. WD3 (1999).

2. V. Fedorov et al., "3.77 - $5.05 \mu$ mtunable solid-state lasers based on $\mathrm{Fe}^{2+}$-doped $\mathrm{ZnSe}$ crystals operating at low and room temperatures," IEEE J. Quantum Electron. 42, 907-917 (2006).

3. V. A. Akimov et al., "Efficient lasing in a $\mathrm{Fe}^{2+}: \mathrm{ZnSe}$ crystal at room temperature," Quantum Electron. 36(4), 299 (2006).

4. A. A. Voronov et al., "A continuous-wave $\mathrm{Fe}^{2+}$ :ZnSe laser," Quantum Electron. 38(12), 1113 (2008).

5. M. E. Doroshenko et al., "Fe:ZnSe laser-comparison of active materials grown by two different methods," Proc. SPIE 7912, 79122D (2011).

6. J. W. Evans, P. A. Berry, and K. L. Schepler, "840 mW continuous-wave Fe:ZnSe laser operating at $4140 \mathrm{~nm}, "$ Opt. Lett. 37, 5021-5023 (2012).

7. M. P. Frolov et al., "Study of a 2J pulsed Fe:ZnSe $4 \mu \mathrm{m}$ laser," Laser Phys. Lett. 10(12), 125001 (2013).

8. J. Evans, P. Berry, and K. Schepler, "A passively Q-switched, CW-pumped Fe:ZnSe laser," IEEE J. Quantum Electron. 50, 204-209 (2014).

9. D. V. Martyshkin et al., "High average power $(35 \mathrm{~W})$ pulsed Fe:ZnSe laser tunable over $3.8-4.2 \mu \mathrm{m}$," in Conf. Lasers and Electro-Opt., p. SF1F.2 (2015).

10. N. Myoung et al., "Energy scaling of $4.3 \mu \mathrm{m}$ room temperature Fe:ZnSe laser," Opt. Lett. 36, 94-96 (2011)

11. J. W. Evans et al., "Optical spectroscopy and modeling of $\mathrm{Fe}^{2+}$ ions in zinc selenide," J. Luminesc. 188, 541-550 (2017).

12. N. F. Mott and R. W. Gurney, Electronic Processes in Ionic Crystals, Dover, New York (1964).

13. J. W. Evans et al., "Re-absorption and nonradiative energy transfer in vibronic laser gain media," Proc. SPIE 10511, 105110I (2018).

14. J. J. Adams et al., "4.0 - 4.5- $\mu$ m lasing of Fe:ZnSe Below $180 \mathrm{~K}$, a new mid-infrared laser material," Opt. Lett. 24, 1720-1722 (1999).

15. Y. Song et al., "Mid-infrared light emission from a $\mathrm{Fe}^{2+}: \mathrm{ZnSe}$ polycrystal using quantum cascade laser pumping," Appl. Phys. Lett. 105(14), 141108 (2014). 
16. L. DeLoach et al., "Transition metal-doped zinc chalcogenides: spectroscopy and laser demonstration of a new class of gain media," IEE J. Quantum Electron. 32, 885-895 (1996).

17. H. Jelínková et al., "Fe:ZnSe laser oscillation under cryogenic and room temperature," Proc. SPIE 8599, 85990E (2013).

18. N. Myoung et al., "Temperature and concentration quenching of mid-IR photoluminescence in iron doped ZnSe and ZnS Laser crystals," J. Luminesc. 132(3), 600-606 (2012).

19. B. Henderson and G. F. Imbusch, Optical Spectroscopy of Inorganic Solids, Monographs on the Physics and Chemistry of Materials, Clarendon Press, Oxford (1989).

20. V. Pagonis et al., "Modelling the thermal quenching mechanism in quartz based on timeresolved optically stimulated luminescence," J. Luminesc. 130(5), 902-909 (2010).

21. T. Miyakawa and D. L. Dexter, "Phonon sidebands, multiphonon relaxation of excited states, and phonon-assisted energy transfer between ions in solids," Phys. Rev. B 1, 2961-2969 (1970).

22. J. W. Evans, R. W. Stites, and T. R. Harris, "Increasing the performance of an Fe:ZnSe laser using a hot isostatic press," Opt. Mater. Express 7, 4296-4303 (2017).

23. A. Martinez, "Spectroscopic characterization of iron-doped II-VI compounds for laser applications," PhD thesis, The University of Alabama at Birmingham (2015).

24. J. B. McKay, "Power scaling feasibility of chromium-doped II-VI laser sources and the demonstration of a chromium-doped zinc selenide face-cooled disk laser," tech. rep., Air Force Institute of Technology (2002).

Jonathan W. Evans is a research physicist at the Air Force Research Laboratory at Wright-Patterson Air Force Base, Ohio. He earned his BS degree in electrical engineering from Cedarville University in 2008. He earned his MS degree in electro-optics from the University of Dayton in 2010 and his PhD in optical sciences and engineering from Air Force Institute of Technology in 2014. His research interests include transition metal lasers, nonlinear parametric conversion, and spectroscopy of novel laser systems.

Thomas R. Harris has worked in the Optoelectronic Technology Branch of the Air Force Research Laboratory, Sensors Directorate at Wright-Patterson Air Force Base since 2015, first as an NRC Postdoctoral research associate and more recently as a contractor with Azimuth Corp. He earned his PhD in applied physics from the Air Force Institute of Technology in 2014. His interests include infrared spectroscopy and transition-metal and rare-earth doped laser host materials.

Eric J. Turner is a laser systems research engineer at KBR. He has experience in solid state laser materials research and development. He earned his BS degree in electrical engineering from Howard University and his MS degree in electro-optics from the University of Dayton.

Kenneth L. Schepler received his BS degree in physics from Michigan State University and MS and $\mathrm{PhD}$ degrees from the University of Michigan. He was a research physicist at the Air Force Research Laboratory (AFRL) at Wright-Patterson Air Force Base for over 32 years. He retired in 2014 and is now a research professor at CREOL. His interests include solid state lasers, laser spectroscopy, and nonlinear frequency conversion. He is a fellow of OSA and AFRL.

Biographies of the other authors are not available. 